\title{
具有高逼近阶和正则性的双向加细函数 和双向小波
}

\author{
杨守志* 李尤发
}

(汕头大学数学系, 汕头 515063 )

摘要引入了双向加细函数和双向小波的概念, 并研究双向加细方程

$$
\phi(x)=\sum_{k} p_{k}^{+} \phi(m x-k)+\sum_{k} p_{k}^{-} \phi(k-m x)
$$

的分布解 (或 $L^{2}$ 稳定解) 的存在性, 其中整数 $m \geqslant 2$. 基于正向面具 $\left\{p_{k}^{+}\right\}$和负向面具 $\left\{p_{k}^{-}\right\}$, 建立了确保双向加细方程具有紧支撑分布解或 $L^{2}$ 稳定解所需要的条件. 更进一步地, 给出 了双向加细方程的 $L^{2}$ 稳定解能产生一个 MRA 所需要的条件. 充分讨论了 $\phi(x)$ 的支撑区 间. 给出正交双向加细函数和双向小波的定义, 建立了双向加细函数的正交准则. 给出一类 正交双向加细函数和正交双向小波的构造算法. 另外, 也给出了具有非负面具的、高逼近阶 和正则性的双向加细函数的构造算法. 最后, 构造了两个算例.

关键词 双向加细函数 双向小波 正交性 逼近阶 正则性

$$
\operatorname{MSC}(2000) \text { 主题分类 } 42 \mathrm{C} 15,94 \mathrm{~A} 12
$$

\section{1 引言}

两尺度加细方程在小波的构造和应用中起着非常重要的作用 (见文献 [1-6]). 众所周知, 除 Haar 小波, 单小波无法同时拥有良好的性质, 诸如紧支撑性、对称性和正交性. 为了避免 单小波的不足, 人们引入多小波的概念, GHM 多小波是能同时具有紧支撑性、对称性和正交 性的一个例子. 近来, 人们已经在多尺度函数和多小波方面做了大量的工作 (见文献 [7-16]). 我们知道, 具有非负面具的尺度向量函数在工程技术方面有很重要应用, 很多人对此做了大 量的研究 (见文献 [17-19]).

本文主要研究如下两尺度双向细方程:

$$
\phi(x)=\sum_{k} p_{k}^{+} \phi(m x-k)+\sum_{k} p_{k}^{-} \phi(k-m x),
$$

给出高逼近阶和正则性的双向加细函数的构造算法. 具体地, 建立了两尺度双向细方程 (1) 具有紧支撑分布解或 $L^{2}$ 稳定解所需要的充分必要条件. 充分讨论了两尺度双向加细方程所 
确定的双向加细函数的支撑区间. 给出两尺度双向细方程的 $L^{2}$ 稳定解能生成一个 MRA 所 需要的条件. 给出正交双向加细函数和对应的正交双向小波的定义; 建立双向加细函数的正 交准则; 并给出一类正交双向加细函数和正交双向小波的构造算法. 讨论了双向加细函数的 逼近阶和正则性, 给出一类具有非负面具、高逼近阶和正则性的双向加细函数的构造算法. 最后提供两个构造算例.

\section{2 双向加细函数}

双向加细函数概念的引入源于基数 B- 样条函数. 称

$$
N_{m}(x)=\int_{-\infty}^{+\infty} N_{m-1}(x-t) N_{1}(t) d t=\int_{0}^{1} N_{m-1}(x-t) d t
$$

是 $m$ 阶基数 B- 样条, 其中 $N_{1}(x)$ 表示区间为 $[0,1)$ 上的特征函数. 根据文献 [6] 知, $N_{m}(x)$ 满足如下的两尺度加细方程:

$$
N_{m}(x)=\sum_{k=0}^{m} p_{k} N_{m}(2 x-k),
$$

其中 $p_{k}=2^{-m+1} \frac{m !}{k !(m-k) !} \cdot N_{m}(x)$ 的支撑区间为 $[0, m)$, 且关于 $\frac{m}{2}$ 点对称.

引理 1 (文献 [7] 的推论 4.1) 假设 $N_{m}(x)$ 是 $m$ 阶基数 B- 样条, 则对任意整数 $\alpha$, 有

$$
N_{m}\left(\alpha^{-1} x\right)=\sum_{k=0}^{m(\alpha-1)} \mu_{m}^{\alpha}(k) N_{m}(x-k),
$$

其中对于一切的 $0 \leqslant \ell<\alpha$, 序列 $\left\{\mu_{m}^{\alpha}(k)\right\}_{k}=\alpha^{-1}\left\{\mu_{m-1}^{\alpha}(j)\right\}_{j} *\left\{\mu_{1}^{\alpha}(\ell)\right\}_{\ell}, \mu_{1}^{\alpha}(\ell)=1$. 此处, * 表示卷积算子.

注 1 根据 (3) 式以及基数 B- 样条的对称性, 得

$$
N_{m}(x)=\sum_{k=0}^{J} \mu_{m}^{\alpha}(k) N_{m}(\alpha x-k)+\sum_{k=J+1}^{m(\alpha-1)} \mu_{m}^{\alpha}(k) N_{m}(-\alpha x+k+m),
$$

这里 $J$ 是任意整数, 并且 $0<J<m(\alpha-1)$. 在这个意义上, 选择合适的序列 $\left\{p_{k}^{+}\right\}_{k}$ 和 $\left\{p_{k}^{-}\right\}_{k}$, 方程 (1) 很可能具有优良解, B- 样条就是一个例证. 事实上, 方程 (1) 是伸缩因子为 $m$ 的加 细函数的一般形式, 因为当所有 $p_{k}^{-}=0$ 时, 它就退化成 $m$ 尺度加细方程.

下面给出方程 (1) 有解的条件, 其中包括紧支撑分布解和 $L^{2}$ 稳定解. 首先, 介绍一些记 号和定义.

假设 $\phi(x)$ 是满足方程 (1) 的双向加细函数. 分别称序列 $\left\{p_{k}^{+}\right\}_{k}$ 及 $\left\{p_{k}^{-}\right\}_{k}$ 为正向面具和 负向面具. 对方程 (1) 的两边进行 Fourier 变换, 可得

$$
\widehat{\phi}(w)=P^{+}\left(e^{-i w / m}\right) \widehat{\phi}\left(\frac{w}{m}\right)+P^{-}\left(e^{-i w / m}\right) \overline{\widehat{\phi}\left(\frac{w}{m}\right)},
$$

其中 $P^{+}(z)=\frac{1}{m} \sum_{k \in Z} p_{k}^{+} z^{k}, P^{-}(z)=\frac{1}{m} \sum_{k \in Z} p_{k}^{-} z^{k}$ 分别是正向面具符号和负向面具符号.

定义 1 称方程 (1) 中定义的 $\phi(x)$ 是 $L^{2}$ 稳定的, 如果存在两个常数 $A$ 和 $B(0<A \leqslant$ $B<\infty)$, 使得

$$
A \sum_{k \in Z} c_{k} c_{k}^{*} \leqslant\left\|\sum_{k \in Z} c_{1}^{k} \phi(x-k)+\sum_{k \in Z} c_{2}^{k} \phi(k-x)\right\|_{2}^{2} \leqslant B \sum_{k \in Z} c_{k} c_{k}^{*} .
$$

其中 $\left\{c_{k}\right\}_{k \in Z}=\left\{\left[c_{1}^{k}, c_{2}^{k}\right]\right\}_{k \in Z}$ 是 $l^{2}(Z)^{2}$ 中的任意一个序列. 
为研究方程 (1) 解的存在性, 对其作变形得

$$
\phi(-x)=\sum_{k} p_{k}^{+} \phi(-m x-k)+\sum_{k} p_{k}^{-} \phi(m x+k) .
$$

对 (5) 式两边施行 Fourier 变换得

$$
\overline{\widehat{\phi}(w)}=\overline{P^{+}\left(e^{-i w / m}\right)} \overline{\widehat{\phi}\left(\frac{w}{m}\right)}+\overline{P^{-}\left(e^{-i w / m}\right)} \widehat{\phi}\left(\frac{w}{m}\right) .
$$

根据 (4) 和 (6) 式, 可得

$$
\left[\begin{array}{l}
\widehat{\phi}(w) \\
\widehat{\widehat{\phi}(w)}
\end{array}\right]=\left[\begin{array}{ll}
P^{+}\left(e^{-i w / m}\right) & P^{-}\left(e^{-i w / m}\right) \\
\overline{P^{-}\left(e^{-i w / m}\right)} & \frac{\widehat{P}+\left(e^{-i w / m}\right)}{P^{+}\left(\frac{w}{m}\right)} \\
\widehat{\widehat{\phi}\left(\frac{w}{m}\right)}
\end{array}\right],
$$

显然, 方程 (1) 有解当且仅当 (7) 式有解.

设

$$
\Phi(x)=\left[\begin{array}{c}
\phi(x) \\
\phi(-x)
\end{array}\right]=\sum_{k \in Z}\left[\begin{array}{cc}
p_{k}^{+} & p_{k}^{-} \\
p_{-k}^{-} & p_{-k}^{+}
\end{array}\right] \Phi(m x-k),
$$

则 (7) 式是 $\Phi(x)$ 在频率域中的 $m$ - 尺度加细方程. 它的加细面具符号是

$$
P(z)=\left[\begin{array}{ll}
P^{+}(z) & P^{-}(z) \\
\overline{P^{-}(z)} & \overline{P^{+}(z)}
\end{array}\right] .
$$

定义 $\phi(x)$ 的自相关矩阵符号

$$
\Omega(w):=\sum_{k}\left[\begin{array}{cc}
\langle\phi(x), \phi(x-k)\rangle & \langle\phi(x), \phi(k-x)\rangle \\
\langle\phi(-x), \phi(x-k)\rangle & \langle\phi(-x), \phi(k-x)\rangle
\end{array}\right] e^{-i k w} .
$$

引入变换算子 $\tau$ :

$$
\tau A\left(e^{-i w}\right)=\sum_{k=0}^{m-1} P\left(\frac{w}{m}+\frac{2 k \pi}{m}\right) A\left(\frac{w}{m}+\frac{2 k \pi}{m}\right) P^{*}\left(\frac{w}{m}+\frac{2 k \pi}{m}\right),
$$

其中 $A\left(e^{-i w}\right)$ 是 Laurent 多项式方阵, $P(w)$ 由 $(9)$ 式给出.

关于 $\Omega(w)$ 和 $\tau$, 我们有如下的引理.

引理 2 矩阵符号 $\Omega(w)$ 和变换算子 $\tau$ 的定义如上, 则由 Poisson 求和公式可得

$$
\begin{aligned}
& \left.\Omega(w)=\sum_{k}\left[\frac{\widehat{\phi}(w+2 k \pi)}{\widehat{\widehat{\phi}(w+2 k \pi)}}\right] \overline{[\widehat{\phi}(w+2 k \pi)} \widehat{\phi}(w+2 k \pi)\right] \\
& =\sum_{k}\left[\begin{array}{ll}
\widehat{\phi}(w+2 k \pi) \overline{\widehat{\phi}(w+2 k \pi)} & \widehat{\phi}(w+2 k \pi) \widehat{\phi}(w+2 k \pi) \\
\overline{\widehat{\phi}(w+2 k \pi) \widehat{\phi}(w+2 k \pi)} & \overline{\widehat{\phi}(w+2 k \pi) \widehat{\phi}(w+2 k \pi)}
\end{array}\right] .
\end{aligned}
$$

更进一步, $\Omega(w)$ 是 $\tau$ 的相应于特征值 1 的特征矩阵, i.e. $\tau \Omega(w)=\Omega(w)$.

证明 设 $\phi_{j}(x)$ 和 $\phi_{\ell}(x)$ 是任意两个函数. 定义

$$
G_{j, k}(y):=\left\langle\phi_{j}(x), \phi_{\ell}(x-y)\right\rangle=\int_{R} \phi_{j}(x) \overline{\phi_{\ell}(x-y)} d x,
$$

则

$$
\sum_{k \in Z}\left\langle\phi_{j}(x), \phi_{\ell}(x-k)\right\rangle e^{-i k w}=\sum_{k \in Z} G_{j, \ell}(k) e^{-i k w} .
$$


令 $F^{-1}$ 代表 Fourier 逆变换, 则由 Poisson 求和公式可得

$$
\sum_{k \in Z} G_{j, \ell}(k) e^{-i k w}=2 \pi \sum_{k \in Z}\left(F^{-1} G_{j, k}\right)(w+2 k \pi) .
$$

另一方面,

$$
\begin{aligned}
\left(F^{-1} G_{j, k}\right)(w) & =\frac{1}{2 \pi} \int_{R}\left(\int_{R} \phi_{j}(x) \overline{\phi_{\ell}(x-y)} d x\right) e^{i w y} d y \\
& =\frac{1}{2 \pi} \int_{R} \phi_{j}(x)\left(\int_{R} \overline{\phi_{\ell}(x-y)} e^{i w y} d y\right) d x \\
& =\frac{1}{2 \pi}\left(\int_{R} \phi_{j}(x) e^{-i w x} d x\right) \overline{\widehat{\phi}_{\ell}(w)} \\
& =\frac{1}{2 \pi} \widehat{\phi}_{j}(w) \overline{\widehat{\phi}_{\ell}(w)} .
\end{aligned}
$$

因此,

$$
\begin{aligned}
\sum_{k \in Z}\left\langle\phi_{j}(x), \phi_{\ell}(x-k)\right\rangle e^{-i k w} & =2 \pi \sum_{k \in Z}\left(F^{-1} G_{j, k}\right)(w+2 k \pi) \\
& =\sum_{k \in Z} \widehat{\phi}_{j}(w+2 k \pi) \overline{\widehat{\phi}_{\ell}(w+2 k \pi)} .
\end{aligned}
$$

从以上的讨论, 很容易得到

$$
\begin{aligned}
& \Omega\left(e^{-i w}\right)=\left[\begin{array}{cc}
\sum_{k \in Z}\langle\phi(x), \phi(x-k)\rangle e^{-i k w} & \sum_{k \in Z}\langle\phi(x), \phi(k-x)\rangle e^{-i k w} \\
\sum_{k \in Z}\langle\phi(-x), \phi(x-k)\rangle e^{-i k w} & \sum_{k \in Z}\langle\phi(-x), \phi(k-x)\rangle e^{-i k w}
\end{array}\right] \\
& =\sum_{k \in Z}\left[\begin{array}{ll}
\widehat{\phi}(w+2 k \pi) \overline{\widehat{\phi}(w+2 k \pi)} & \widehat{\phi}(w+2 k \pi) \widehat{\phi}(w+2 k \pi) \\
\overline{\widehat{\phi}(w+2 k \pi) \widehat{\phi}(w+2 k \pi)} & \overline{\widehat{\phi}(w+2 k \pi)} \widehat{\phi}(w+2 k \pi)
\end{array}\right] \\
& =\sum_{k \in Z} \Phi(w+2 k \pi) \Phi^{*}(w+2 k \pi) \text {. }
\end{aligned}
$$

因此,

$$
\begin{aligned}
\tau \Omega(m w)= & \sum_{k=0}^{m-1} P\left(w+\frac{2 k \pi}{m}\right) \Omega\left(w+\frac{2 k \pi}{m}\right) P^{*}\left(w+\frac{2 k \pi}{m}\right) \\
= & \sum_{k=0}^{m-1} \sum_{\ell \in Z} P\left(w+2 \ell \pi+\frac{2 k \pi}{m}\right) \Phi\left(w+2 \ell \pi+\frac{2 k \pi}{m}\right) \\
& \times \Phi^{*}\left(w+2 \ell \pi+\frac{2 k \pi}{m}\right) P^{*}\left(w+2 \ell \pi+\frac{2 k \pi}{m}\right) \\
= & \sum_{k=0}^{m-1} \sum_{\ell \in Z} \Phi(m w+2 \ell m \pi+2 k \pi) \Phi^{*}(m w+2 \ell m \pi+2 k \pi) \\
= & \sum_{k=0}^{m-1} \sum_{\ell \in Z} \Phi(m w+2 \pi(\ell m+k)) \Phi^{*}(m w+2 \pi(\ell m+k)) \\
= & \sum_{\ell^{\prime} \in Z} \Phi\left(m w+2 \ell^{\prime} \pi\right) \Phi^{*}\left(m w+2 \ell^{\prime} \pi\right)=\Omega(m w) .
\end{aligned}
$$


定理 1 加细方程 (1) 存在一个紧支撑分布解当且仅当
(I) $\left\{\begin{array}{l}\sum_{k}\left(p_{k}^{+}-p_{k}^{-}\right)=m, \\ \left|\sum_{k}\left(p_{k}^{+}+p_{k}^{-}\right)\right| \leqslant m,\end{array}\right.$
或
$\left\{\begin{array}{l}\sum_{k}\left(p_{k}^{+}+p_{k}^{-}\right)=m, \\ \left|\sum_{k}\left(p_{k}^{+}-p_{k}^{-}\right)\right| \leqslant m .\end{array}\right.$

证明 根据文献 [8-11], 方程 (8) 存在紧支撑分布解当且仅当 1 是 $(9)$ 式定义的矩阵 $P(1)$ 的特征值, $P(1)$ 的其他特征值的模不大于 1 . 另一方面, $P(1)$ 的两个特征值是 $\frac{1}{m} \sum_{k}\left(p_{k}^{+}-p_{k}^{-}\right)$ 和 $\frac{1}{m} \sum_{k}\left(p_{k}^{+}+p_{k}^{-}\right)$. 定理易证.

定理 2 加细方程 (1) 只有一个紧支撑分布解当且仅当

$$
\text { (III) }\left\{\begin{array} { l } 
{ \sum _ { k } ( p _ { k } ^ { + } - p _ { k } ^ { - } ) = m ， } \\
{ | \sum _ { k } ( p _ { k } ^ { + } + p _ { k } ^ { - } ) | < m ， }
\end{array} \text { 或 (IV) } \left\{\begin{array}{l}
\sum_{k}\left(p_{k}^{+}+p_{k}^{-}\right)=m ， \\
\left|\sum_{k}\left(p_{k}^{+}-p_{k}^{-}\right)\right|<m .
\end{array}\right.\right.
$$

证明 根据文献 [8-11], 方程 (8) 只有一个紧支撑分布解当且仅当 1 是 (9) 式定义的矩 阵 $P(1)$ 的一个单特征值, 其余的特征值的模严格小于 1 . 另一方面, $P(1)$ 的两个特征值是 $\frac{1}{m} \sum_{k}\left(p_{k}^{+}-p_{k}^{-}\right)$和 $\frac{1}{m} \sum_{k}\left(p_{k}^{+}+p_{k}^{-}\right)$. 定理易证.

根据引理 2 和文献 $[11,12]$, 容易得到以下的定理:

定理 3 双向加细函数 $\phi(x)$ 是 $L^{2}$ - 稳定的当且仅当它满足如下的条件: $P(z)$ 满足定理 2 中的条件; (10) 式定义的变换算子 $\tau$ 满足条件 $E$, 它的特征值 1 对应的特征矩阵 $\Omega(w)$ 是正 定的, $\forall w \in \mathbb{R}$.

\section{3 双向加细函数的支撑区间}

本节研究双向加细函数的支撑区间.

设 $\Phi(x)=\left(\phi_{1}(x), \phi_{2}(x), \ldots, \phi_{r}(x)\right)^{\mathrm{T}}$ 是伸缩因子为 $m$ 的多尺度函数, 满足加细方程

$$
\Phi(x)=\sum_{k=0}^{N} P_{k} \Phi(m x-k) .
$$

定义 $\Phi(x)$ 的矩阵符号 $P(z):=\frac{1}{m} \sum_{k=0}^{N} P_{k} z^{k}$, 它是 $r \times r$ 的多项式矩阵.

本节使用如下的记号: $h(i, j)$ 表示矩阵 $P(z)$ 的 $(i, j)$ 元素的最高次数, $l(i, j)$ 表示 $(i, j)$ 元 素的最低次数. $I_{k}$ 代表 $k$ 价单位矩阵; $e_{k}$ 代表单位矩阵的第 $k$ 列, 它的维数可从上下文得知. $E_{\ell, k}$ 表示 $e_{\ell} e_{k}^{\mathrm{T}}$. 令 $\Omega$ 表示所有整数序列 $J=\left(j_{1}, \ldots, j_{r}\right)$ 的集合, 其中 $1 \leqslant j_{1}, \ldots, j_{r} \leqslant r$. 对于 每个 $J=\left(j_{1}, \ldots, j_{r}\right) \in \Omega$, 定义 $E_{J}=m I_{r}-E_{1 j_{1}}-\cdots-E_{r j_{r}}, h_{J}=\left[h\left(1, j_{1}\right), \ldots, h\left(r, j_{r}\right)\right]^{\mathrm{T}}, l_{J}=$ $\left[l\left(1, j_{1}\right), \ldots, l\left(r, j_{r}\right)\right]^{\mathrm{T}}$. 注意到 $E_{J}$ 是可逆的并且所有的元素均为非负数, 更进一步地 $E_{J}^{-1}=$ $\sum_{k=0}^{\infty} \frac{1}{m^{k+1}} E^{k}$, 其中 $E=E_{1 j_{1}}+\cdots+E_{r j_{r}}$.

下面给出引理 3, 类似文献 [20] 的定理 2.1 的证明方法可以证明引理 3 .

引理 3 设 $\Phi(x)=\left(\phi_{1}(x), \phi_{2}(x), \ldots, \phi_{r}(x)\right)^{\mathrm{T}}$ 是伸缩因子为 $m$ 的多尺度函数, 它满足方 程 (11). 假定 $\phi_{i}(x)$ 的支撑区间是 $\left[L_{i}, R_{i}\right]$, 则

$$
R_{i} \leqslant \max \left\{e_{i}^{\mathrm{T}} E_{J}^{-1} h_{J}: J \in \Omega\right\}, \quad L_{i} \geqslant \min \left\{e_{i}^{\mathrm{T}} E_{J}^{-1} l_{J}: J \in \Omega\right\} .
$$

引理 4 假设多尺度函数 $\Phi(x)=\left(\phi_{1}(x), \phi_{2}(x), \ldots, \phi_{r}(x)\right)^{\mathrm{T}}$ 满足加细方程: $\Phi(x)=$ $\sum_{k=-n}^{N} C_{k} \Phi(m x-k)$, 其中 $C_{-n}, C_{N} \neq 0$. 令 $\widetilde{\Phi}(x)=\Phi\left(x+\frac{n}{m-1}\right)$, 则 $\widetilde{\Phi}(x)$ 也是多尺度函 
数且满足加细方程

$$
\widetilde{\Phi}(x)=\sum_{k=0}^{N+n} C_{k-n} \widetilde{\Phi}(m x-k) .
$$

定理 4 设双向加细函数 $\phi(x)$ 满足加细方程:

$$
\phi(x)=\sum_{k=0}^{N} p_{k}^{+} \phi(m x-k)+\sum_{k=-N}^{0} p_{k}^{-} \phi(k-m x) .
$$

如果两序列 $\left\{p_{k}^{+}\right\}$和 $\left\{p_{k}^{-}\right\}$满足定理 2 的条件, 则 $\operatorname{Supp} \phi(x) \subseteq\left[-\frac{N}{m-1}, \frac{N}{m-1}\right]$.

证明 因为两序列 $\left\{p_{k}^{+}\right\}$和 $\left\{p_{k}^{-}\right\}$满足定理 2 的条件, 可以定义一个多尺度函数 $\Phi(x)=$ $(\phi(x), \phi(-x))^{\mathrm{T}}$, 则 $\Phi(x)$ 满足加细方程

$$
\Phi(x)=\sum_{k=-N}^{N} P_{k} \Phi(m x-k)=\sum_{k=-N}^{N}\left[\begin{array}{cc}
p_{k}^{+} & p_{k}^{-} \\
p_{-k}^{-} & p_{-k}^{+}
\end{array}\right] \Phi(m x-k) .
$$

定义 $\widetilde{\Phi}(x):=\left(\widetilde{\phi}_{1}(x), \widetilde{\phi}_{2}(x)\right)^{\mathrm{T}}=\Phi\left(x+\frac{N}{m-1}\right)$, 则根据引理 $4, \widetilde{\Phi}(x)$ 满足加细方程

$$
\widetilde{\Phi}(x)=\sum_{k=0}^{2 N} P_{k-N} \widetilde{\Phi}(m x-k) .
$$

$\widetilde{\Phi}(x)$ 的矩阵符号是 $\widetilde{P}(z)=\frac{1}{m} \sum_{k=0}^{2 N} P_{k-N} z^{k}$. 根据引理 3 和 4 , 可得

$\operatorname{Supp} \widetilde{\phi}_{1}(x)=\operatorname{Supp} \phi\left(x+\frac{N}{m-1}\right)=\left[L_{1}, R_{1}\right], \quad \operatorname{Supp} \widetilde{\phi}_{2}(x)=\operatorname{Supp} \phi\left(-x+\frac{N}{m-1}\right)=\left[L_{2}, R_{2}\right]$,

其中 $R_{i} \leqslant \max \left\{e_{i}^{\mathrm{T}} E_{J}^{-1} h_{J}: J \in \Omega\right\}, L_{i} \geqslant \min \left\{e_{i}^{\mathrm{T}} E_{J}^{-1} l_{J}: J \in \Omega\right\}, i=1,2$. 换言之,

$$
B_{1} \leqslant x+\frac{N}{m-1} \leqslant A_{1}, \quad B_{2} \leqslant-x+\frac{N}{m-1} \leqslant A_{2},
$$

其中 $A_{i}=\max \left\{e_{i}^{\mathrm{T}} E_{J}^{-1} h_{J}: J \in \Omega\right\}, B_{i}=\min \left\{e_{i}^{\mathrm{T}} E_{J}^{-1} l_{J}: J \in \Omega\right\}$. 此处的 $E_{J}, e_{i}, h_{J}, l_{J}$ 如同引 理 3 中定义. 因此,

$\operatorname{Supp} \phi(x) \subseteq\left[\max \left(B_{1}-\frac{N}{m-1}, \frac{N}{m-1}-A_{2}\right), \quad \min \left(A_{1}-\frac{N}{m-1}, \frac{N}{m-1}-B_{2}\right)\right]$. 令

$$
\begin{aligned}
& E_{J_{1}}=m I_{2}-E_{11}-E_{21}=\left[\begin{array}{cc}
m-1 & 0 \\
-1 & m
\end{array}\right], \quad E_{J_{2}}=m I_{2}-E_{11}-E_{22}=\left[\begin{array}{cc}
m-1 & 0 \\
0 & m-1
\end{array}\right], \\
& E_{J_{3}}=m I_{2}-E_{12}-E_{22}=\left[\begin{array}{cc}
m & -1 \\
0 & m-1
\end{array}\right], \quad E_{J_{4}}=m I_{2}-E_{12}-E_{21}=\left[\begin{array}{cc}
m & -1 \\
-1 & m
\end{array}\right] .
\end{aligned}
$$

易知

$$
\begin{aligned}
& h(1,1)=2 N, \quad h(1,2)=N, \quad h(2,1)=2 N, \quad h(2,2)=N, \\
& l(1,1)=N, \quad l(1,2)=0, \quad l(2,1)=N, \quad l(2,2)=0 .
\end{aligned}
$$

因此,

$$
\begin{cases}h_{J_{1}}=[h(1,1), h(2,1)]^{\mathrm{T}}=[2 N, 2 N]^{\mathrm{T}} & l_{J_{1}}=[l(1,1), l(2,1)]^{\mathrm{T}}=[N, N]^{\mathrm{T}}, \\ h_{J_{2}}=[h(1,1), h(2,2)]^{\mathrm{T}}=[2 N, N]^{\mathrm{T}} & l_{J_{2}}=[l(1,1), l(2,2)]^{\mathrm{T}}=[N, 0]^{\mathrm{T}}, \\ h_{J_{3}}=[h(1,2), h(2,2)]^{\mathrm{T}}=[N, N]^{\mathrm{T}} & l_{J_{3}}=[l(1,2), l(2,2)]^{\mathrm{T}}=[0,0]^{\mathrm{T}}, \\ h_{J_{4}}=[h(1,2), h(2,1)]^{\mathrm{T}}=[N, 2 N]^{\mathrm{T}} & l_{J_{4}}=[l(1,2), l(2,1)]^{\mathrm{T}}=[0, N]^{\mathrm{T}} .\end{cases}
$$


所以从 (12) 式, 可得 $A_{1}=A_{2}=\frac{2 N}{m-1}, B_{1}=B_{2}=0$. 根据 (13) 式, 可知 $\operatorname{Supp} \phi(x) \subseteq\left[-\frac{N}{m-1}\right.$, $\frac{N}{m-1}$ ]. 定理 4 得证.

\section{4 双向多分辨分析}

定义子空间序列 $\left\{V_{j}\right\} \subset L^{2}(R)$,

$$
V_{j}=\operatorname{Clos}_{L^{2}(R)}\left\langle m^{\frac{j}{2}} \phi\left(m^{j} x-k, m^{\frac{j}{2}} \phi\left(l-m^{j} x\right), k, l \in \mathbb{Z}\right\rangle .\right.
$$

像往常一样, 方程 (1) 中定义的 $\phi(x)$ 能产生一个 $L^{2}(R)$ 中的多分辨分析 $\left\{V_{j}\right\}_{j \in Z}$ 当且仅当 (15) 式中定义的 $\left\{V_{j}\right\}$ 满足
(i) $\cdots \subset V_{-1} \subset V_{0} \subset V_{1} \subset \cdots$;
(ii) $\operatorname{Clos}_{L^{2}(R)}\left(\bigcup_{j \in Z} V_{j}\right)=L^{2}(R)$;
(iii) $\bigcap_{j \in Z}\left\{V_{j}\right\}=\{0\}$;
(iv) $f(x) \in V_{j} \Longleftrightarrow f(m x) \in V_{j+1}$;

(v) 集合 $\{\phi(x-k), \phi(n-x): k, n \in Z\}$ 是 $V_{0}$ 的 Riesz 基.

具体地说, 性质 (v) 意味着存在正常数 $A \leqslant B<\infty$, 使得对于系数向量序列 $\left\{\mathbf{c}_{\ell}\right\}=$ $\left\{\left[c_{1}^{\ell}, c_{2}^{\ell}\right]\right\}_{\ell \in Z} \in \ell^{2}(Z)^{2}$ 有

$$
A \sum_{\ell \in Z} c_{\ell}^{*} c_{\ell} \leqslant\left\|\sum_{\ell \in Z} c_{1}^{\ell} \phi(x-\ell)+c_{2}^{\ell} \phi(\ell-x)\right\|_{L^{2}}^{2} \leqslant B \sum_{\ell \in Z} c_{\ell}^{*} c_{\ell} .
$$

定义 $\phi_{j, k}^{+}(x):=m^{j / 2} \phi\left(2^{j} x-k\right), \phi_{j, k}^{-}(x):=m^{j / 2} \phi\left(k-2^{j} x\right)$. 由多分辨分析的定义可知, $\left\{\phi_{j, k}^{+}(x), \phi_{j, k}^{-}(x): k \in Z\right\}$ 也构成 $V_{j}$ 的 Riesz 基, 即

$$
A \sum_{\ell \in Z} c_{\ell}^{*} c_{\ell} \leqslant\left\|\sum_{\ell \in Z} c_{1}^{\ell} \phi_{j, \ell}^{+}(x)+c_{2}^{\ell} \phi_{j, k}^{-}(x)\right\|_{L^{2}}^{2} \leqslant B \sum_{\ell \in Z} c_{\ell}^{*} c_{\ell} .
$$

因为 $\phi(x) \in V_{0} \subset V_{1}$ 且 $\left\{\phi_{1, k}^{+}(x), \phi_{1, k}^{-}(x): k \in Z\right\}$ 构成 $V_{1}$ 的 Riesz 基, 所以存在两个序 列 $\left\{p_{k}^{+}\right\} \in \ell^{2}$ 和 $\left\{p_{k}^{-}\right\} \in \ell^{2}$, 使得 $\phi(x)$ 满足 (1).

定理 5 假设 $\phi(x) \in L^{2}(R)$ 满足 $(1)$, 定义 $V_{j}:=\operatorname{Clos}_{L^{2}(R)}\left\langle\phi_{j, k}^{+}(x), \phi_{j, k}^{-}(x), k \in Z\right\rangle$. 如果 集合 $\{\phi(x-k), \phi(n-x): k, n \in Z\}$ 构成 $V_{0}$ 的 Riesz 基, 则 $\bigcap_{j \in Z} V_{j}=\{0\}$.

证明 因为 $\left\{\phi_{0, k}^{+}(x), \phi_{0, k}^{-}(x), k \in Z\right\}$ 构成 $V_{0}$ 的 Riesz 基, 所以存在两个常数 $A \leqslant B<\infty$, 使得对于任意的 $f(x) \in V_{0}$, 都有

$$
A\|f\|^{2} \leqslant \sum_{k \in Z}\left[\left|\left\langle f, \phi_{0, k}^{+}\right\rangle\right|^{2}+\left|\left\langle f, \phi_{0, k}^{-}\right\rangle\right|^{2}\right] \leqslant B\|f\|^{2},
$$

因此, 对所有 $f(x) \in V_{j}$, 有

$$
A\|f\|^{2} \leqslant \sum_{k \in Z}\left[\left|\left\langle f, \phi_{j, k}^{+}\right\rangle\right|^{2}+\left|\left\langle f, \phi_{j, k}^{-}\right\rangle\right|^{2}\right] \leqslant B\|f\|^{2} .
$$

现在取 $f(x) \in \bigcap_{j \in Z} V_{j}, \varepsilon>0$ 任意小. 存在一个紧支撑连续的函数 $\tilde{f}$, 使得 $\|f-\tilde{f}\|_{L^{2}} \leqslant \varepsilon$. 假 定 $P_{j}$ 是 $V_{j}$ 上的正交投影算子, 则

$$
\left\|f-P_{j} \tilde{f}\right\|=\left\|f-P_{j} \widetilde{f}\right\| \leqslant\|f-\widetilde{f}\| \leqslant \varepsilon .
$$

因此,

$$
\|f\| \leqslant \varepsilon+\left\|P_{j} \widetilde{f}\right\|, \quad \forall j \in Z
$$


假设 $\operatorname{Supp} \widetilde{f}(x)=[-R, R]$, 则

$$
\begin{aligned}
\sum_{k \in Z}\left[\left|\left\langle\widetilde{f}, \phi_{j, k}^{+}\right\rangle\right|^{2}\right. & \leqslant m^{j} \sum_{k \in Z}\left[\int_{|x| \leqslant R}\left|\widetilde{f}(x) \| \phi\left(m^{j} x-k\right)\right| d x\right]^{2} \\
& \leqslant m^{j}\|\widetilde{f}\|_{L^{\infty}}^{2} \sum_{k \in Z}\left[\int_{|x| \leqslant R}\left|\phi\left(m^{j} x-k\right)\right| d x\right]^{2} \\
& \leqslant m^{j}\|\widetilde{f}\|_{L^{\infty}}^{2} \sum_{k \in Z} \int_{|x| \leqslant R}\left|\phi\left(m^{j} x-k\right)\right|^{2} d x \\
& =2 R\|\widetilde{f}\|_{L^{\infty}}^{2} \int_{S_{j}}|\phi(y)|^{2} d y,
\end{aligned}
$$

其中 $S_{j}=\bigcup_{k \in Z}\left[k-m^{j} R, k+m^{j} R\right]$. 因此, 可证 $\lim _{j \rightarrow-\infty} \sum_{k \in Z}\left[\left|\left\langle\tilde{f}, \phi_{j, k}^{+}\right\rangle\right|^{2}=0\right.$. 同理可证, $\lim _{j \rightarrow-\infty} \sum_{k \in Z}\left[\left|\left\langle\tilde{f}, \phi_{j, k}^{-}\right\rangle\right|^{2}=0\right.$. 又因为 $\left\|P_{j} \tilde{f}\right\| \leqslant A^{-1 / 2}\left[\sum_{k \in Z}\left[\left|\left\langle f, \phi_{j, k}^{+}\right\rangle\right|^{2}+\left|\left\langle f, \phi_{j, k}^{-}\right\rangle\right|^{2}\right]\right]^{1 / 2}$, 所 以 $\lim _{j \rightarrow-\infty}\left\|P_{j} \widetilde{f}\right\|=0$. 最后根据 (20) 式, 可得 $f(x)=0$. 定理得证.

定理 6 假设 $\phi(x) \in L^{2}(R)$ 满足 $(1)$. 定义 $V_{j}:=\operatorname{Clos}_{L^{2}(R)}\left\langle\phi_{j, k}^{+}(x), \phi_{j, k}^{-}(x), k \in \mathbb{Z}\right\rangle$. 如果 $\phi(x)$ 满足 (a) 集合 $\left\{\phi_{0, k}^{+}(x), \phi_{0, k}^{-}(x), k \in Z\right\}$ 是 $V_{0}$ 的 Resiz 基; (b) 对于所有 $w \in R, \widehat{\phi}(w)$ 有 界; (c) $\widehat{\phi}(w)$ 在 $w=0$ 附近连续并且 $\widehat{\phi}(0) \neq 0$, 则 $\operatorname{Clos}_{L^{2}(R)}\left(\bigcup_{j \in Z} V_{j}\right)=L^{2}(R)$.

证明 因为 $\left\{\phi_{0, k}^{+}(x), \phi_{0, k}^{-}(x), k \in Z\right\}$ 构成 $V_{0}$ 的 Riesz 基, 存在两个常数 $A \leqslant B<\infty$, 使 得对所有 $f(x) \in V_{0},(17)$ 式成立, 因此, 对所有 $f(x) \in V_{j},(18)$ 式成立.

取 $f(x) \in\left(\bigcap_{j \in Z} V_{j}\right)^{\perp}, \varepsilon>0$ 任意小. 存在一个紧支撑连续的函数 $\tilde{f} \in C^{\infty}$, 使得 $\| f-$ $\widetilde{f} \|_{L^{2}(R)} \leqslant \varepsilon$.

因此, 对一切 $J \in Z$, 有 $\left\|P_{J} \tilde{f}\right\|=\left\|P_{J}(\tilde{f}-f)\right\| \leqslant \varepsilon$. 又因为 $P_{J} \tilde{f} \in V_{J}$, 则

$$
\left\|P_{J} \widetilde{f}\right\|^{2} \geqslant B^{-1} \sum_{k \in Z}\left[\left|\left\langle\widetilde{f}, \phi_{J, k}^{+}\right\rangle\right|^{2}+\left|\left\langle\tilde{f}, \phi_{J, k}^{-}\right\rangle\right|^{2}\right] .
$$

进一步推导可得

$$
\sum_{k \in Z}\left[\left|\left\langle\widetilde{f}, \phi_{J, k}^{+}\right\rangle\right|^{2}+\left|\left\langle\tilde{f}, \phi_{J, k}^{-}\right\rangle\right|^{2}\right]=2 \pi \int_{-\infty}^{\infty}\left[\left|\widehat{\phi}\left(m^{J} w\right)\right|^{2}+\left|\overline{\widehat{\phi}\left(m^{J} w\right)}\right|^{2}\right]|\widehat{\widetilde{f}}(w)|^{2} d w+E,
$$

其中

$$
E \leqslant\left(\|\widehat{\phi}\|_{L^{\infty}}^{2}+\|\overline{\widehat{\phi}}\|_{L^{\infty}}^{2}\right) \sum_{\ell \neq 0} \int_{-\infty}^{\infty}\left|\widehat{\widetilde{f}}(w) \| \widehat{\widetilde{f}}\left(w+m^{-J} 2 \pi \ell\right)\right| d w .
$$

因为 $\tilde{f} \in C^{\infty}$, 所以存在一个常数 $C$, 使得

$$
|\widehat{\widetilde{f}}(w)| \leqslant C\left(1+|w|^{2}\right)^{-3 / 2} .
$$

因此,

$$
\begin{aligned}
E & \leqslant C^{2}\left(\|\widehat{\phi}\|_{L^{\infty}}^{2}+\|\overline{\widehat{\phi}}\|_{L^{\infty}}^{2}\right) \sum_{\ell \neq 0} \int_{-\infty}^{\infty}\left(1+\left|w+m^{-J} \pi \ell\right|^{2}\right)^{-3 / 2}\left(1+\left|w-m^{-J} \pi \ell\right|^{2}\right)^{-3 / 2} d w \\
& \leqslant C^{2}\left(\|\widehat{\phi}\|_{L^{\infty}}^{2}+\|\overline{\widehat{\phi}}\|_{L^{\infty}}^{2}\right) \sum_{\ell \neq 0}\left(1+\pi^{2} \ell^{2} m^{-2 J}\right)^{-1 / 2} \int_{-\infty}^{\infty}\left(1+|w|^{2}\right)^{-1} d w \\
& \leqslant C^{\prime} m^{J} .
\end{aligned}
$$

综合以上的推导, 可得

$2 \pi \int_{-\infty}^{\infty}\left[\left|\widehat{\phi}\left(m^{J} w\right)\right|^{2}+\left|\overline{\widehat{\phi}\left(m^{J} w\right)}\right|^{2}\right]|\widehat{\widetilde{f}}(w)|^{2} d w \leqslant \sum_{k \in Z}\left[\left|\left\langle\widetilde{f}, \phi_{J, k}^{+}\right\rangle\right|^{2}+\left|\left\langle\widetilde{f}, \phi_{J, k}^{-}\right\rangle\right|^{2}\right]+|E| \leqslant B \varepsilon^{2}+C^{\prime} m^{J}$. 
因为 $\widehat{\phi}(w)$ 一致有界且在 $w=0$ 处连续, 所以当 $J \rightarrow-\infty$ 时, 上面不等式的左边收玫于 $2 \pi\left[|\widehat{\phi}(0)|^{2}+|\overline{\widehat{\phi}(0)}|^{2}\right]\|\widetilde{f}\|_{L^{2}}^{2}$. 由此可得

$$
\|\widetilde{f}\|_{L^{2}} \leqslant \frac{\sqrt{B} \varepsilon}{2 \pi \sqrt{\left[|\widehat{\phi}(0)|^{2}+|\widehat{\widehat{\phi}(0) \mid}|^{2}\right]}} .
$$

所以,

$$
\|f\|_{L^{2}} \leqslant \varepsilon+\|\widetilde{f}\|_{L^{2}} \leqslant \varepsilon+\frac{\sqrt{B} \varepsilon}{2 \pi \sqrt{\left[|\widehat{\phi}(0)|^{2}+|\widehat{\widehat{\phi}(0)}|^{2}\right]}} \leqslant\left(1+\frac{\sqrt{B}}{2 \pi \sqrt{\left[|\widehat{\phi}(0)|^{2}+|\widehat{\widehat{\phi}(0)}|^{2}\right]}}\right) \varepsilon .
$$

因为 $\varepsilon$ 任意小, 所以 $f=0$. 这表明了 $\operatorname{Clos}_{L^{2}(R)}\left(\bigcup_{j \in Z} V_{j}\right)=L^{2}(R)$.

\section{5 正交双向加细函数}

本节定义正交双向加细函数以及相应的正交双向小波. 给出了正交双向加细函数和正交 双向小波的构造算法.

定义 2 设 $\phi(x)$ 是方程 (1) 中定义的伸缩因子为 $m$ 的双向加细函数. 如果它满足

$$
\langle\phi(x), \phi(x-k)\rangle=\delta_{0, k}, \quad\langle\phi(x), \phi(n-x)\rangle=0,
$$

则称 $\phi(x)$ 是正交双向加细函数.

定理 7 设 $\phi(x)$ 是正交双向加细函数, $P^{+}(z)$ 和 $P^{-}(z)$ 分别是它的正向面具及负向面 具, 则

$$
\left\{\begin{array}{l}
\sum_{k=0}^{m-1}\left|P^{+}\left(e^{-i\left(w+\frac{2 k \pi}{m}\right)}\right)\right|^{2}+\sum_{k=0}^{m-1}\left|P^{-}\left(e^{-i\left(w+\frac{2 k \pi}{m}\right)}\right)\right|^{2}=1, \\
\sum_{k=0}^{m-1} P^{+}\left(e^{-i\left(w+\frac{2 k \pi}{m}\right)}\right) P^{-}\left(e^{-i\left(w+\frac{2 k \pi}{m}\right)}\right)=0 .
\end{array}\right.
$$

证明 显然 $\phi(x)$ 正交当且仅当 (8) 式中定义的 $\Phi(x)$ 正交. 因此 $(9)$ 式中定义的 $P(z)$ 满足完全重构条件

$$
\sum_{k=0}^{m-1} P\left(e^{-i\left(w+\frac{2 k \pi}{m}\right)}\right) P^{*}\left(e^{-i\left(w+\frac{2 k \pi}{m}\right)}\right)=I_{2}
$$

即

$$
\left\{\begin{array}{l}
\sum_{k=0}^{m-1}\left|P^{+}\left(e^{-i\left(w+\frac{2 k \pi}{m}\right)}\right)\right|^{2}+\sum_{k=0}^{m-1}\left|P^{-}\left(e^{-i\left(w+\frac{2 k \pi}{m}\right)}\right)\right|^{2}=1, \\
\sum_{k=0}^{m-1} P^{+}\left(e^{-i\left(w+\frac{2 k \pi}{m}\right)}\right) P^{-}\left(e^{-i\left(w+\frac{2 k \pi}{m}\right)}\right)=0 .
\end{array}\right.
$$

设 $\phi(x)$ 是正交双向加细函数, 对任意 $j \in Z$, 定义 $V_{j}$ 在 $V_{j+1}$ 中的正交补 $W_{j}$. 即 $V_{j+1}=V_{j} \oplus W_{j}$. 因此, 对于 $j \neq k$, 都有 $W_{j} \perp W_{k}$ 且 $L^{2}(R)=\bigoplus_{j \in Z} W_{j}$. 如果存在函数 $\psi^{\ell}(x), \ell=1, \ldots, m-1$, 使得集合 $\left\{\psi^{\ell}(x-k), \psi^{\ell}(k-x), k \in Z, \ell=1, \ldots, m-1\right\}$ 构成 $W_{0}$ 的 正交基, 则 $\psi^{\ell}(x)$ 是 $\phi(x)$ 的正交双向小波, 因此, $\psi^{\ell}(x)$ 必须满足

$$
\left\{\begin{array}{l}
\left\langle\phi(x), \psi^{\ell}(x-k)\right\rangle=0,\left\langle\phi(x), \psi^{\ell}(k-x)\right\rangle=0, \\
\left\langle\psi^{\ell}(x-k), \psi^{n}(x)\right\rangle=\delta_{n, \ell} \delta_{k, 0},\left\langle\psi^{\ell}(k-x), \psi^{n}(x)\right\rangle=0 .
\end{array}\right.
$$


假设双向加细函数 $\phi(x)$ 的双向小波 $\psi^{\ell}(x)$ 满足

$$
\psi^{\ell}(x)=\sum_{k} q_{k, \ell}^{+} \phi(m x-k)+\sum_{k} q_{k, \ell}^{-} \phi(k-m x), \quad \ell=1, \ldots, m-1 .
$$

对 $(25)$ 式两边施行 Fourier 变换, 可得

$$
\widehat{\psi}^{\ell}(w)=Q_{\ell}^{+}\left(e^{-i w / m}\right) \widehat{\phi}\left(\frac{w}{m}\right)+Q_{\ell}^{-}\left(e^{-i w / m}\right) \overline{\widehat{\phi}\left(\frac{w}{m}\right)},
$$

其中 $Q_{\ell}^{+}(z)=\frac{1}{m} \sum_{k \in Z} q_{k, \ell}^{+} z^{k}$ 和 $Q_{\ell}^{-}(z)=\frac{1}{m} \sum_{k \in Z} q_{k, \ell}^{-} z^{k}$ 分别称为 $\psi^{\ell}(x)$ 的正向符号及负向 符号.

下面给出正交双向加细函数和相应的正交双向小波的构造算法.

定理 8 假设 $p(w)$ 和 $q_{j}(w)$ 分别是某个伸缩因子为 $m$ 的正交尺度函数及其正交小波 的面具, $j=1,2, \ldots, m-1$. 构造

$$
\left\{\begin{array}{l}
P^{+}(z)=\frac{1}{m} \sum_{k \in Z} p_{k}^{+} z^{k}=\lambda_{0}(w) p(w)+\sum_{i=1}^{m-1} \lambda_{i}(w) q_{i}(w) \\
P^{-}(z)=\frac{1}{m} \sum_{k \in Z} p_{k}^{-} z^{k}=\lambda_{m}(w) p(w)+\sum_{i=1}^{m-1} \lambda_{m+i}(w) q_{i}(w)
\end{array}\right.
$$

假定 $\lambda_{i}(w)(i=0,1, \ldots, 2 m-1)$ 是周期为 $\frac{2 \pi}{m}$ 的周期函数且满足

$$
\left\{\begin{array}{l}
\sum_{i=0}^{2 m-1}\left|\lambda_{i}(w)\right|^{2}=1 \\
\sum_{i=0}^{m-1} \lambda_{i}(w) \overline{\lambda_{m+i}(w)}=0 \\
\lambda_{0}(0)-\lambda_{m}(0)=1 \\
\left|\lambda_{0}(0)+\lambda_{m}(0)\right|<1
\end{array}\right.
$$

或

$$
\left\{\begin{array}{l}
\sum_{i=0}^{2 m-1}\left|\lambda_{i}(w)\right|^{2}=1 \\
\sum_{i=0}^{m-1} \lambda_{i}(w) \overline{\lambda_{m+i}(w)}=0 \\
\lambda_{0}(0)+\lambda_{m}(0)=1 \\
\left|\lambda_{0}(0)-\lambda_{m}(0)\right|<1
\end{array}\right.
$$

则 $P^{+}(z)$ 和 $P^{-}(z)$ 能产生一个正交双向加细函数 $\phi(x)$, 即 $\phi(x)$ 满足以下双向加细方程:

$$
\phi(x)=\sum_{k} p_{k}^{+} \phi(m x-k)+\sum_{k} p_{k}^{-} \phi(k-m x) .
$$


证明 令

$$
\begin{aligned}
P(z) & =\left[\begin{array}{ll}
P^{+}(z) & P^{-}(z) \\
\overline{P^{-}(z)} & \overline{P^{+}(z)}
\end{array}\right] \\
= & {\left[\begin{array}{cc}
\lambda_{0}(w) p(w)+\sum_{i=1}^{m-1} \lambda_{i}(w) q_{i}(w) & \lambda_{m}(w) p(w)+\sum_{i=1}^{m-1} \lambda_{m+i}(w) q_{i}(w) \\
\lambda_{m}(w) p(w)+\sum_{i=1}^{m-1} \lambda_{m+i}(w) q_{i}(w) & \lambda_{0}(w) p(w)+\sum_{i=1}^{m-1} \lambda_{i}(w) q_{i}(w)
\end{array}\right] . }
\end{aligned}
$$

容易证明 $\sum_{k=0}^{m-1} P\left(e^{-i\left(w+\frac{2 k \pi}{m}\right)}\right) P^{*}\left(e^{-i\left(w+\frac{2 k \pi}{m}\right)}\right)=I_{2}$, 即

$$
\left\{\begin{array}{l}
\sum_{k=0}^{m-1}\left|P^{+}\left(e^{-i\left(w+\frac{2 k \pi}{m}\right)}\right)\right|^{2}+\sum_{k=0}^{m-1}\left|P^{-}\left(e^{-i\left(w+\frac{2 k \pi}{m}\right)}\right)\right|^{2}=1, \\
\sum_{k=0}^{m-1} P^{+}\left(e^{-i\left(w+\frac{2 k \pi}{m}\right)}\right) P^{-}\left(e^{-i\left(w+\frac{2 k \pi}{m}\right)}\right)=0 .
\end{array}\right.
$$

另一方面, $P(1)$ 的两个特征值是 $\lambda_{0}(0)-\lambda_{m}(0)$ 和 $\lambda_{0}(0)+\lambda_{m}(0)$, 因此, $P(1)$ 满足条件 E. 根 据定理 2 和 7 , 方程 $(29)$ 存在一个正交解.

推论 如果定理 8 中的 $\lambda_{i}(w)(i=0,1, \ldots, 2 m-1)$ 均为常数, 则当 $m=2$ 时, 方程 $(27)$ 的通解是

$$
\left\{\begin{array}{l}
\lambda_{0}=\frac{b+1}{2}, \\
\lambda_{1}= \pm \frac{\sqrt{1-b^{2}}}{2}, \\
\lambda_{2}=\frac{b-1}{2}, \\
\lambda_{3}= \pm \frac{\sqrt{1-b^{2}}}{2} .
\end{array}\right.
$$

当 $m=3$ 时, 方程 $(27)$ 的通解是

$$
\left\{\begin{array}{l}
\lambda_{2}= \pm \frac{1}{2} \sqrt{1-\left[\left(\lambda_{0}+\lambda_{3}\right)^{2}+\left(\lambda_{1}+\lambda_{4}\right)^{2}\right]} \pm \frac{1}{2} \sqrt{1-\left(\lambda_{0}-\lambda_{3}\right)^{2}}, \\
\lambda_{0}=\frac{b+1}{2} \\
\lambda_{3}=\frac{b-1}{2} \\
\lambda_{5}= \pm \frac{1}{2} \sqrt{1-\left[\left(\lambda_{0}+\lambda_{3}\right)^{2}+\left(\lambda_{1}+\lambda_{4}\right)^{2}\right]} \mp \frac{1}{2} \sqrt{1-\left(\lambda_{0}-\lambda_{3}\right)^{2}} .
\end{array}\right.
$$

构造 $Q_{\ell}^{+}(z)$ 和 $Q_{\ell}^{-}(z)(\ell=1,2, \ldots, m-1)$

$$
\left\{\begin{aligned}
Q_{\ell}^{+}(z)=\frac{1}{m} \sum_{k \in Z} q_{k, \ell}^{+} z^{k} & =\mu_{0}^{\ell}(w) p(w)+\sum_{i=1}^{m-1} \mu_{i}^{\ell}(w) q_{i}(w), \\
Q_{\ell}^{-}(z)=\frac{1}{m} \sum_{k \in Z} q_{k, \ell}^{-} z^{k} & =\mu_{m}^{\ell}(w) p(w)+\sum_{i=1}^{m-1} \mu_{m+i}^{\ell}(w) q_{i}(w) .
\end{aligned}\right.
$$

定理 9 在定理 8 条件下, 如果 $\mu_{i}^{\ell}(w)(i=0,1, \ldots, 2 m-1 ; \ell=1,2, \ldots, m-1)$ 是 $\frac{2 \pi}{m}$ 周 
期的周期函数, 且满足

$$
\left\{\begin{array}{l}
\sum_{i=0}^{m-1}\left[\lambda_{i}(w) \bar{\mu}_{i}^{\ell}(w)+\bar{\lambda}_{m+i}(w) \mu_{m+i}^{\ell}(w)\right]=0 \\
\sum_{i=0}^{m-1}\left[\lambda_{i}(w) \bar{\mu}_{m+i}^{\ell}(w)+\bar{\lambda}_{m+i}(w) \mu_{i}^{\ell}(w)\right]=0, \\
\sum_{i=0}^{m-1}\left[\mu_{i}^{\ell^{\prime}}(w) \bar{\mu}_{i}^{\ell}(w)+\bar{\mu}_{m+i}^{\ell^{\prime}}(w) \mu_{m+i}^{\ell}(w)\right]=\delta_{\ell, \ell^{\prime}}, \\
\sum_{i=0}^{m-1}\left[\mu_{i}^{\ell^{\prime}}(w) \bar{\mu}_{m+i}^{\ell}(w)+\bar{\mu}_{m+i}^{\ell^{\prime}}(w) \mu_{i}^{\ell}(w)\right]=0,2, \ldots, m-1,
\end{array}\right.
$$

则 $\phi(x)$ 的正交双向小波 $\psi^{\ell}(x)(\ell=1,2, \ldots, m-1)$ 可通过符号 $Q_{\ell}^{+}(z)$ 和 $Q_{\ell}^{-}(z)$ 构造, $\ell=$ $1,2, \ldots, m-1$, 即

$$
\psi^{\ell}(x)=\sum_{k} q_{k, \ell}^{+} \phi(m x-k)+\sum_{k} q_{k, \ell}^{-} \phi(k-m x), \quad \ell=1,2, \ldots, m-1 .
$$

证明 令 $\Phi(x)=(\phi(x), \phi(-x))^{\mathrm{T}}$, 则 $\Phi(x)$ 是一正交多尺度函数. 根据 (9) 式可知它的加 细面具是

$$
P(z)=\left[\begin{array}{ll}
P^{+}(z) & P^{-}(z) \\
\overline{P^{-}(z)} & \overline{P^{+}(z)}
\end{array}\right]
$$

设 $\Psi^{\ell}(x)=\left(\psi^{\ell}(x), \psi^{\ell}(-x)\right)^{\mathrm{T}}, \ell=1,2, \ldots, m-1$, 显然, 它相对于 $\Phi(x)$ 的加细面具是

$$
Q^{\ell}(z)=\left[\begin{array}{ll}
Q_{\ell}^{+}(z) & Q_{\ell}^{-}(z) \\
\hline Q_{\ell}^{-}(z) & Q_{\ell}^{+}(z)
\end{array}\right]
$$

$\psi^{\ell}(x)(\ell=1,2, \ldots, m-1)$ 是 $\phi(x)$ 的双向正交小波当且仅当 $\Psi^{\ell}(x)$ 是 $\Phi(x)$ 的正交多小波. 另 一方面, 易证

$$
\sum_{k=0}^{m-1} P\left(e^{-i\left(w+\frac{2 k \pi}{m}\right)}\right) Q^{\ell}\left(e^{-i\left(w+\frac{2 k \pi}{m}\right)}\right)^{*}=O, \quad \sum_{k=0}^{m-1} Q^{\ell^{\prime}}\left(e^{-i\left(w+\frac{2 k \pi}{m}\right)}\right) Q^{\ell}\left(e^{-i\left(w+\frac{2 k \pi}{m}\right)}\right)^{*}=\delta_{\ell, \ell^{\prime}} I,
$$

因此定理得证.

\section{6 双向加细函数的逼近阶}

定义 3 伸缩因子为 $m$ 的双向加细函数 $\phi(x)$ 具有逼近阶 $N$, 如果多项式 $t^{j}(j=0,1, \ldots$, $N-1)$ 是 $\phi(x)$ 和 $\phi(-x)$ 的整数平移的线性组合, 即

$$
t^{j}=\sum_{k} a_{k}^{j} \phi(x-k)+\sum_{k} b_{k}^{j} \phi(k-x) .
$$

定理 10 设 $\phi(x)$ 是满足方程 (1) 的双向加细函数, 则 $\phi(x)$ 提供 $N$ 逼近阶当且仅当 $P^{+}(z)$ 和 $P^{-}(z)$ 满足以下条件: 存在向量 $u_{k}=\left(u_{k}^{1}, u_{k}^{2}\right), u_{0} \neq 0(k=0,1, \ldots, N-1)$, 使得对 
于 $n=0,1, \ldots, N-1$ 和 $r=1,2, \ldots, m-1$ 有

$$
\left\{\begin{array}{l}
\sum_{k=0}^{n} \sum_{\ell}\left(\begin{array}{l}
n \\
k
\end{array}\right)\left(-\frac{\ell}{m}\right)^{n-k}\left(u_{k}^{1} p_{\ell}^{+}+u_{k}^{2} p_{-\ell}^{-}\right)=m^{-n+1} u_{n}^{1}, \\
\sum_{k=0}^{n} \sum_{\ell}\left(\begin{array}{l}
n \\
k
\end{array}\right)\left(-\frac{\ell}{m}\right)^{n-k}\left(u_{k}^{1} p_{\ell}^{-}+u_{k}^{2} p_{-\ell}^{+}\right)=m^{-n+1} u_{n}^{2}, \\
\sum_{k=0}^{n} \sum_{\ell}\left(\begin{array}{l}
n \\
k
\end{array}\right)\left(-\frac{\ell}{m}\right)^{n-k}\left(u_{k}^{1} p_{\ell}^{+}+u_{k}^{2} p_{-\ell}^{-}\right) e^{-i \frac{2 \pi r \ell}{m}}=0, \\
\sum_{k=0}^{n} \sum_{\ell}\left(\begin{array}{l}
n \\
k
\end{array}\right)\left(-\frac{\ell}{m}\right)^{n-k}\left(u_{k}^{1} p_{\ell}^{-}+u_{k}^{2} p_{-\ell}^{+}\right) e^{-i \frac{2 \pi r \ell}{m}}=0 .
\end{array}\right.
$$

证明 令 $\Phi(x)=(\phi(x), \phi(-x))^{\mathrm{T}}$. 它满足方程 $(8)$ 和 $(9) . \phi(x)$ 具有逼近阶 $m$ 当且仅当 $\Phi(x)$ 具有逼近阶 $m$. 根据文献 [12] 的定理 3.1.1, $\Phi(x)$ 具有逼近阶 $m$, 当且仅当方程 $(9)$ 中定 义的加细面具 $P(w)$ 满足: 存在向量 $u_{k}=\left(u_{k}^{1}, u_{k}^{2}\right) \in R^{2} ; u_{0} \neq 0(k=0,1, \ldots, N-1)$, 使得对 于 $n=0,1, \ldots, N-1$, 有

$$
\begin{gathered}
\sum_{k=0}^{n}\left(\begin{array}{l}
n \\
k
\end{array}\right) u_{k}(m i)^{k-n}\left(D^{n-k} P\right)(0)=m^{-n} u_{n} \\
\sum_{k=0}^{n}\left(\begin{array}{l}
n \\
k
\end{array}\right) u_{k}(m i)^{k-n}\left(D^{n-k} P\right)\left(\frac{2 \pi r}{m}\right)=0 .
\end{gathered}
$$

以上条件等价于 $(34)$ 式.

下面给出一类具有高逼近阶、正则性和 $L^{2}$ - 稳定的双向加细函数构造.

定理 11 假定 $\phi(x)$ 是双向加细函数, 它的正向面具符号是 $P^{+}(z)=\frac{1}{m} \sum_{k \in Z} p_{k}^{+} z^{k}=$ $\left(\frac{1+z+\cdots+z^{m-1}}{m}\right)^{M} S^{+}(z)$, 负向面具符号是 $P^{-}(z)=\frac{1}{m} \sum_{k \in Z} p_{k}^{-} z^{k}=\left(\frac{1+z+\cdots+z^{m-1}}{m}\right)^{L} S^{-}(z)$. 此 处的 $S^{+}(z)=\sum_{k \in Z} s_{k}^{+} z^{k}, S^{-}(z)=\sum_{k \in Z} s_{k}^{-} z^{k}, s_{k}^{+} \geqslant 0, s_{k}^{-} \geqslant 0$. 进一步地, 假设以下条件成 立:

$$
\begin{cases}S^{+}(1)+S^{-}(1)=1, & \\ S^{-}\left(e^{\frac{-i 2(m-1) \pi}{m}}\right) \neq 0, & \text { 当 } M>L, \\ S^{+}\left(e^{\frac{-i 2(m-1) \pi}{m}}\right) \neq 0, & \text { 当 } L>M, \\ S^{+}\left(e^{\frac{-i 2(m-1) \pi}{m}}\right)+e^{-\frac{i 2 \pi L}{m}} S^{-}\left(e^{-\frac{i 2(m-1) \pi}{m}}\right) \neq 0, & \text { 当 } L=M,\end{cases}
$$

则 $\phi(x)$ 属于 $L^{2}(R)$ 稳定的, 并且具有 $n$ 阶逼近阶, 正则数不少于 $n-\frac{1}{2}$, 其中 $n=\min (M, L)$.

证明 令 $\Phi(x)=(\phi(x), \phi(-x))^{\mathrm{T}}$, 则 $\phi(x)$ 有 $m$ 阶逼近阶当且仅当 $\Phi(x)$ 有 $m$ 阶逼近阶. 根据 (7) 式, $\Phi(x)$ 的加细面具是

$$
P(z)=\left[\begin{array}{ll}
P^{+}(z) & P^{-}(z) \\
\overline{P^{-}(z)} & \overline{P^{+}(z)}
\end{array}\right] .
$$

假定 $M \geqslant L$, 则

$$
\begin{aligned}
P(z)= & \left(\frac{1+z+\cdots+z^{m-1}}{m}\right)^{L} \\
& \times\left[\begin{array}{cc}
\left(\frac{1+z+\cdots+z^{m-1}}{m}\right)^{M-L} S^{+}(z) & S^{-}(z) \\
z^{-L} \frac{S^{-}(z)}{m} & z^{-M}\left(\frac{1+z+\cdots+z^{m-1}}{m-L} \overline{S^{+}(z)}\right.
\end{array}\right] .
\end{aligned}
$$


$P(1)$ 的两个特征值是 $S^{+}(1)+S^{-}(1)$ 和 $S^{+}(1)-S^{-}(1)$. 因为 $s_{k}^{+} \geqslant 0, s_{k}^{-} \geqslant 0, S^{+}(1)+S^{-}(1)=1$, 所以 $\left|S^{+}(1)-S^{-}(1)\right|<1$, 因此 $P(z)$ 满足条件 E. 根据定理 2 , 方程 (1) 存在唯一解. 定义

$$
\begin{aligned}
H(z) & :=\left(h_{\ell j}(z)\right) \\
& =\left[\begin{array}{cc}
\left(\frac{1+z+\cdots+z^{m-1}}{m}\right)^{M-L} S^{+}(z) & S^{-}(z) \\
z^{-L} \overline{S^{-}(z)} & z^{-M}\left(\frac{1+z+\cdots+z^{m-1}}{m}\right)^{M-L} \overline{S^{+}(z)}
\end{array}\right] .
\end{aligned}
$$

$[1,1] H(1)=[1,1]$, 然而 $[1,1] H\left(e^{-\frac{i 2(m-1) \pi}{m}}\right) \neq[0,0]$, 因此 $H(z)$ 提供 0 阶逼近阶. 根据文献 $[12]$ 的引理 3.3.7 可知 $\Phi(x)$ 具有 $m$ 阶逼近阶, 即 $\phi(x)$ 具有 $m$ 阶逼近阶. 定义

$$
\begin{aligned}
& H_{n}\left(e^{-i w}\right)=\left(H_{n}(\ell, j)\left(e^{-i w}\right)\right):=\prod_{k=1}^{n} H\left(e^{-i \frac{w}{m^{k}}}\right), \\
& P_{n}\left(e^{-i w}\right):=\prod_{k=1}^{n} P\left(e^{-i \frac{w}{m^{k}}}\right)=\prod_{k=1}^{n}\left(\frac{1+e^{-i \frac{w}{m^{k}}}+\cdots+e^{-i \frac{(m-1) w}{m^{k}}}}{m}\right)^{L} \times H_{n}\left(e^{-i w}\right), \\
& H_{\infty}\left(e^{-i w}\right)=\left(H_{\infty}(\ell, j)\left(e^{-i w}\right)\right):=\prod_{k=1}^{\infty} H\left(e^{-i \frac{w}{m^{k}}}\right) .
\end{aligned}
$$

因为 $s_{k}^{+} \geqslant 0$ 且 $s_{k}^{-} \geqslant 0$, 所以

$$
\begin{aligned}
& \max _{|z|=1}\left|\left(\frac{1+z+\cdots+z^{m-1}}{m}\right)^{M-L} S^{+}(z)\right|=S^{+}(1), \quad \max _{|z|=1}\left|S^{-}(z)\right|=S^{-}(1), \\
& \max _{|z|=1}\left|z^{-M}\left(\frac{1+z+\cdots+z^{m-1}}{m}\right)^{M-L} \overline{S^{+}(z)}\right|=S^{+}(1), \quad \max _{|z|=1}\left|z^{-L} \overline{S^{-}(z)}\right|=S^{-}(1) .
\end{aligned}
$$

换言之，

$$
\left|h_{11}(z)\right| \leqslant S^{+}(1), \quad\left|h_{22}(z)\right| \leqslant S^{+}(1), \quad\left|h_{12}(z)\right| \leqslant S^{-}(1), \quad\left|h_{21}(z)\right| \leqslant S^{-}(1) .
$$

另一方面,

$$
H_{2}\left(e^{-i w}\right)=\prod_{k=1}^{2} H\left(e^{-i \frac{w}{m^{k}}}\right)=\left[\begin{array}{ll}
\left(H_{2}(1,1)\right)\left(e^{-i w}\right) & \left(H_{2}(1,2)\right)\left(e^{-i w}\right) \\
\left(H_{2}(2,1)\right)\left(e^{-i w}\right) & \left(H_{2}(2,2)\right)\left(e^{-i w}\right)
\end{array}\right],
$$

其中

$$
\begin{aligned}
& \left(H_{2}(1,1)\right)\left(e^{-i w}\right)=h_{11}\left(e^{-i w / m}\right) h_{11}\left(e^{-i w / m^{2}}\right)+h_{12}\left(e^{-i w / m}\right) h_{12}\left(e^{-i w / m^{2}}\right), \\
& \left(H_{2}(1,2)\right)\left(e^{-i w}\right)=h_{11}\left(e^{-i w / m}\right) h_{12}\left(e^{-i w / m^{2}}\right)+h_{12}\left(e^{-i w / m}\right) \overline{h_{11}\left(e^{-i w / m^{2}}\right)}, \\
& \left(H_{2}(2,1)\right)\left(e^{-i w}\right)=\overline{h_{11}\left(e^{-i w / m}\right) h_{12}\left(e^{-i w / m^{2}}\right)}+\overline{h_{12}\left(e^{-i w / m}\right)} h_{11}\left(e^{-i w / m^{2}}\right), \\
& \left(H_{2}(2,2)\right)\left(e^{-i w}\right)=\overline{h_{11}\left(e^{-i w / m}\right) h_{11}\left(e^{-i w / m^{2}}\right)+h_{12}\left(e^{-i w / m}\right) h_{12}\left(e^{-i w / m^{2}}\right)} .
\end{aligned}
$$

根据 (37) 和 (38) 式可知

$$
\begin{aligned}
& \left|h_{11}\left(e^{-i w / m}\right) h_{11}\left(e^{-i w / m^{2}}\right)+h_{12}\left(e^{-i w / m}\right) h_{12}\left(e^{-i w / m^{2}}\right)\right| \leqslant\left(S^{+}(1)\right)^{2}+\left(S^{-}(1)\right)^{2}, \\
& \left|h_{11}\left(e^{-i w / m}\right) h_{12}\left(e^{-i w / m^{2}}\right)+h_{12}\left(e^{-i w / m}\right) \overline{h_{11}\left(e^{-i w / m^{2}}\right)}\right| \leqslant 2 S^{+}(1) S^{-}(1) .
\end{aligned}
$$

当 $w=0$, 以上两个不等式变成等式, 进一步地,

$$
h_{11}^{2}(1)+h_{12}^{2}(1)=\left(S^{+}(1)\right)^{2}+\left(S^{-}(1)\right)^{2}, \quad h_{11}(1) h_{12}(1)=S^{+}(1) S^{-}(1),
$$


即 $\left(H_{2}(1,1)\right)(1)+\left(H_{2}(1,2)\right)(1)=1$. 对 $n$ 作数学归纳法可得

$$
\begin{aligned}
& \left|\left(H_{\infty}(1,1)\right)\left(e^{-i w}\right)\right| \leqslant\left(H_{\infty}(1,1)\right)(1)<1, \quad\left|\left(H_{\infty}(2,2)\right)\left(e^{-i w}\right)\right|=\left|\overline{\left(H_{\infty}(1,1)\right)\left(e^{-i w}\right)}\right|, \\
& \left|\left(H_{\infty}(1,2)\right)\left(e^{-i w}\right)\right| \leqslant\left(H_{\infty}(1,2)\right)(1)<1, \quad\left|\left(H_{\infty}(2,1)\right)\left(e^{-i w}\right)\right|=\left|\overline{\left(H_{\infty}(1,2)\right)\left(e^{-i w}\right)}\right|, \\
& \left(H_{\infty}(1,1)\right)(1)+\left(H_{\infty}(1,2)\right)(1)=1 .
\end{aligned}
$$

因为 $[1,1]^{\mathrm{T}}$ 是 $P(1)$ 的特征值 1 对应的特征向量, 并且根据 (7) 式, 可得

$$
\begin{aligned}
& {\left[\frac{\widehat{\phi}(w)}{\widehat{\phi}(w)}\right]=\prod_{k=1}^{\infty}\left[\begin{array}{ll}
P^{+}\left(e^{-i w / m^{k}}\right) & P^{-}\left(e^{-i w / m^{k}}\right) \\
P^{-}\left(e^{-i w / m^{k}}\right) & \frac{P^{+}\left(e^{-i w / m^{k}}\right)}{}
\end{array}\right] \times\left[\begin{array}{l}
1 \\
1
\end{array}\right]} \\
& =\prod_{k=1}^{\infty}\left(\frac{1+e^{-i \frac{w}{m^{k}}}+\cdots+e^{-i \frac{(m-1) w}{m^{k}}}}{m}\right)^{L} \\
& \times \prod_{k=1}^{\infty}\left[\frac{h_{11}\left(e^{-i w / m^{k}}\right)}{h_{12}\left(e^{-i w / m^{k}}\right)} \frac{h_{12}\left(e^{-i w / m^{k}}\right)}{h_{11}\left(e^{-i w / m^{k}}\right)}\right] \times\left[\begin{array}{l}
1 \\
1
\end{array}\right] .
\end{aligned}
$$

根据 (39)-(42) 式, 可得

$$
\begin{aligned}
|\widehat{\phi}(w)| & =\left|\prod_{k=1}^{\infty}\left(\frac{1+e^{-i \frac{w}{m^{k}}}+\cdots+e^{-i \frac{(m-1) w}{m^{k}}}}{m}\right)^{L}\right|\left|\left(H_{\infty}(11)\right)\left(e^{-i w}\right)+\left(H_{\infty}(12)\right)\left(e^{-i w}\right)\right| \\
& \leqslant\left|\prod_{k=1}^{\infty}\left(\frac{1+e^{-i \frac{w}{m^{k}}}+\cdots+e^{-i \frac{(m-1) w}{m^{k}}}}{m}\right)^{L}\right| .
\end{aligned}
$$

设 $N_{L}(x)$ 表示伸缩因子为 $m$ 的 $L$ 阶 B- 样条尺度函数. 根据引理 1 , 它的加细面具是 $\left(\frac{1+z+\cdots+z^{m-1}}{m}\right)^{L}$, 即

$$
\widehat{N}_{L}(w)=\prod_{k=1}^{\infty}\left(\frac{1+e^{-i \frac{w}{m^{k}}}+\cdots+e^{-i \frac{(m-1) w}{m^{k}}}}{m}\right)^{L} .
$$

因为 $\widehat{N}_{m}(w) \in L^{2}(R)$, 所以由 $(43)$ 式可知, $\widehat{\phi}(w) \in L^{2}(R)$. 根据 Fourier 变换等距同构可知 $\phi(x) \in L^{2}(R)$.

众所周知, $L$ 阶 B- 样条尺度函数 $N_{L}(x)$ 具有正则数 $L-\frac{1}{2}$, 即

$$
\int\left|\widehat{N}_{L}(w)\right|^{2}\left(1+|w|^{2}\right)^{L-\frac{1}{2}} d w<\infty .
$$

根据 (43) 和 (44) 式, 可得 $\int|\widehat{\phi}(w)|^{2}\left(1+|w|^{2}\right)^{L-\frac{1}{2}} d w<\infty$, 因此 $\phi(x)$ 的正则数不少于 $L-\frac{1}{2}$. 对于 $M \leqslant L$ 的情形可类似讨论.

注 2 在定理 11 , 选取合适的 $n$, 可得到期望的逼近阶和正则性.

\section{7 算例}

例 $\mathbf{1}(m=2$ 的情形 $)$ 设 $\phi(x)$ 是满足方程 (1) 的双向加细函数. 它的正向面具和负向 面具分别是

$$
P^{+}(z)=\left(\frac{1+z}{2}\right)^{3} \frac{z^{2}}{2(1+\alpha)}, \quad P^{-}(z)=\left(\frac{1+z}{2}\right)^{2}\left[\frac{z}{2}+\frac{\alpha}{2(1+\alpha)}\right],
$$

其中 $\alpha>0$. 根据定理 $5, \phi(x) \in L^{2}(R)$ 具有 2 阶逼近阶, 正则数不小于 1.50 . 如果 $\alpha=0.225$, 
则

$$
\begin{aligned}
\phi(x)= & 0.102 \phi(2 x-2)+0.3062 \phi(2 x-3)+0.3062 \phi(2 x-4)+0.102 \phi(2 x-5) \\
& +0.0460 \phi(-2 x)+0.3418 \phi(-2 x-1)+0.546 \phi(-2 x-2)+0.25 \phi(-2 x-3) .
\end{aligned}
$$

利用文献 [21] 中的 Matlab 程序, 发现 $\phi \in C^{1.9499}$, 其中 $C^{\gamma}$ 表示 Sobolev 光滑. 我们作 了 $\phi(x)$ 的图形 (如图 1).

例 $\mathbf{2}(m=3$ 的情形 $)$ 设 $\phi(x)$ 是满足方程 $(1)$ 的双向加细函数. 它的正方向面具和负 方向面具分别是

$$
P^{+}(z)=\left(\frac{1+z+z^{2}}{3}\right)^{3} \frac{1}{2(1+\alpha)}, \quad P^{-}(z)=\left(\frac{1+z+z^{2}}{3}\right)^{2}\left[\frac{z}{2}+\frac{\alpha}{2(1+\alpha)}\right],
$$

其中 $\alpha>0$. 根据定理 $5, \phi(x) \in L^{2}(R)$ 提供 2 阶逼近阶, 它的正则数不小于 1.50 . 如果取 $\alpha=0.0225$, 则

$$
\begin{aligned}
\phi(x)= & 0.0543 \phi(3 x)+0.1629 \phi(3 x-1)+0.3261 \phi(3 x-2)+0.3804 \phi(3 x-3)+0.3261 \phi(3 x-4) \\
& +0.1629 \phi(3 x-5)+0.0543 \phi(3 x-6)+0.0036 \phi(-3 x)+0.1740 \phi(-3 x-1) \\
& +0.3444 \phi(-3 x-2)+0.5073 \phi(-3 x-3)+0.3369 \phi(-3 x-4)+0.1668 \phi(-3 x-5) .
\end{aligned}
$$

用文献 [21] 中的 Matlab 程序, 发现 $\phi \in C^{1.9528}$. 我们作了 $\phi(x)$ 的图形 (如图 2).

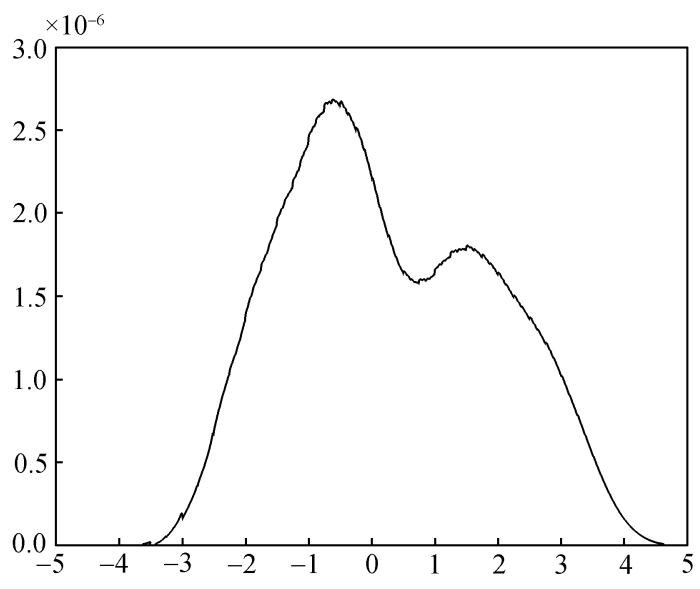

图 1 例 1 中 $\phi(x)$ 的图形

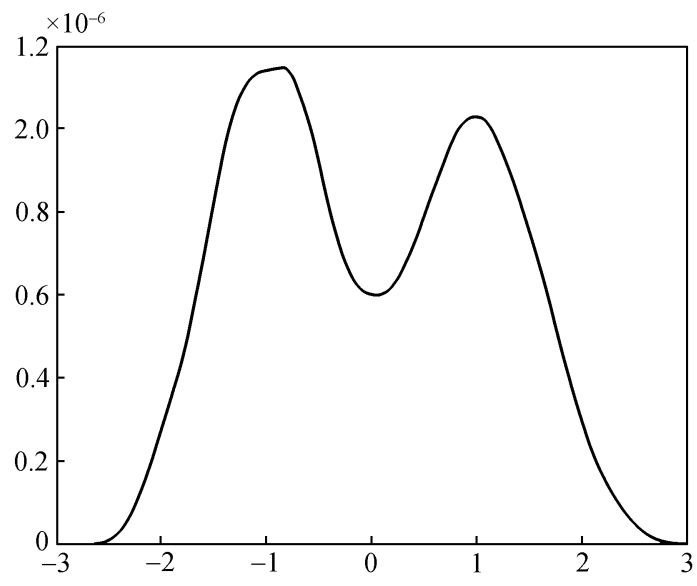

图 2 例 2 中 $\phi(x)$ 的图形

致谢 感谢审稿人对初稿提出的宝贵修改意见和建议, 使文章有了较大的改进; 在此也 感谢与 Lian Jian-ao 教授有益的讨论.

\section{参考 文 献}

1 Daubechies I. Ten Lectures on Wavelets, Ten Lectureson Wavelets. CBM-SNSF Series in Applied Math $\sharp$ 61. Philadelphia: SIAM Publ, 1992

2 Daubechies I. Orthonormal basis of compactly supported wavelet. Comm Pure and Appl Math, 41(7): 909-996 (1988)

3 Chui C K, Wang J Z. On compactly supported spline wavelets and a duality principle. Trans Amer Math Soc, 330(2): 903-915 (1992) 
4 Daubechies I, Lagarias J C. Two-scale difference equation I: Existence and global regularity of solutions. SIAM J Math Anal, 22(5): 1388-1410 (1991)

5 Daubechies I, Lagarias J C. Two-scale difference equation II: Local regularity, infinite products of matrices and fractal. SIAM J Math Anal, 23(4): 1031-1079 (1992)

6 Chui C K. An introduction to wavelet. New York: Academic Press, 1992

7 Nelson J D B. A wavelet filter enhancement scheme with a fast integral B-wavelet transform and pyramidal multi-B-wavelet algorithm. Appl Comput Hamon Anal, 18(3): 234-251 (2005)

8 Jiang Q T. Parameterization of $M$-channel orthogonal multiwavelets banks. Adv Comp Math, 12(2-3): 189-211 (2000)

9 Shen Z W. Refinable function vectors. SIAM J Math Anal, 29(1): 235-250 (1998)

10 Cabrelli C, Heil C, Molter U. Accuracy of lattice translation of several multidimensional refinable function. J Approx Theory, 95(1): 5-52 (1998)

11 Lagarias J C, Wang Y. Orthogonality criteria for compactly supported refinable functions and refinable vectors. J Fourier Anal Appl, 6(2): 153-170 (2000)

12 Plonka G, Strela V. From wavelets to multiwavelets. Mathematical Methods for Curves and Surfaces II, 1998

13 杨守志, 彭立中. 基于重数延长法提升加细向量函数的逼近阶. 中国科学 A 辑: 数学, 2005, 35(12): 13471360

14 Yang S Z, Cheng Z X, Wang H Y. Construction of biorthogonal multiwavelets. J Math Anal Appl, 276(1): 1-12 (2002)

15 Yang S Z. A fast algorithm for constructing orthogonal multiwavelets. ANZIAM J, 46(2): 185-202 (2004)

16 Lian J A. Orthogonality criteria for multi-scaling funtions. Appl Comput Hamon Anal, 5(3): 277-311 (1998)

17 Melkman A A. Subdivision schemes with nonnegative masks always converge-unless they obviously cannot? Ann Numer Math, 4(1-4): 451-460 (1997)

18 Wang Y. Subdivision schemes and refinement equations with nonnegative masks. J Approx Theory, 113(2): 207-220 (2001)

19 Zhou X L. Subdivision schemes with nonnegative masks. Math Comp, 74(250): 819-839 (2005)

20 Wasin S, Wang J Z. Estimating the support of a scaling vector. SIAM J Matrix Anal Appl, 1(1): 66-73 (1997)

21 Jiang Q T. Matlab routines for Sobolev and Hölder smoothness computation of refinable functions. Available: http://www.cs.umsl.edu/ jiang/Jsofware.htm, 2001 